\section{Adenine Phosphoribosyltransferase Deficiency in a Chinese Man with Early-onset Gout}

\section{To the Editor:}

Early-onset gout is known to be often related to genetic factors. However, the molecular genetic issues are not understood for these patients.

In 2007, a 25-year-old Hong Kong Chinese man was referred for advice on his early-onset gout. The first of his many attacks was at age 18 years. A severe attack appeared at his right first metatarsophalangeal joint when he was 22 years old. His serum uric acid then was $600 \mu \mathrm{mol} / \mathrm{l}(10 \mathrm{mg} / \mathrm{dl})$ and serum creatinine was $1.1 \mathrm{mg} / \mathrm{l}$. His creatinine clearance was about 80 $\mathrm{cc} / \mathrm{min} 3$ years ago compared to $88 \mathrm{cc} / \mathrm{min} 10$ years ago. He had 24-h uric acid excretion (using a low purine diet) at $5625 \mu \mathrm{mol} / \mathrm{day}(940 \mathrm{mg} / \mathrm{day}$ ), indicating overproduction of uric acid.

The treatment of choice for gout with overproduction of uric acid is allopurinol, but the patient was worried about the safety of allopurinol because of his allergic history to other drugs and his father's allergy to allopurinol. We suggested that the test of HLA-B $* 5801$ might be helpful. The HLA-B*5801 allele was reported in one study to be present in all 51 $(100 \%)$ Chinese patients with allopurinol-related severe cutaneous adverse reactions, but in only 20 (15\%) of 135 allopurinol-tolerant patients (odds ratio 580.3$)^{1}$. His HLA-B typing was $* 1302 / * 5401$. Therefore, there was very little or no risk of allopurinol allergy for him. Thus, colchicine $0.5 \mathrm{mg}$ daily plus allopurinol $100-300 \mathrm{mg}$ daily was suggested to prevent gout attacks and renal stone formation.

To search for a cause of the overproduction, hypoxanthine-guanine phosphoribosyltransferase (HGPRT) and adenine phosphoribosyltransferase (APRT) activity were measured by high performance liquid chromatography ${ }^{2}$. HGPRT activity was in the normal range. To our surprise, his APRT activity was as low as $0.11 \mu \mathrm{mol} / \mathrm{min}$ per $\mathrm{g}$ hemoglobin (normal range $0.32-0.449$ ). These data suggested that his gout might be related to partial deficiency of APRT, a very rare situation in a Chinese population. Allopurinol was also felt to be appropriate to treat this deficiency ${ }^{3,4}$. After 1 year and 2 months, he is taking allopurinol $250 \mathrm{mg}$ daily and has been free of gout attack; his uric acid concentration is $5.7 \mathrm{mg} / \mathrm{dl}(338 \mu \mathrm{mol} / \mathrm{l})$.

In further investigations, genomic DNA was extracted from buffy coats using a QIAmp DNA blood kit (Qiagen, Valencia, CA, USA). Polymerase chain reaction (PCR) was performed with the following primers; forward GCA TCG ACT ACA TCG CAG, and reverse CTG CAG GAG AGA GAA GAA G. PCR-DNA sequencing revealed a heterozygote mutation $(\mathrm{G} \rightarrow \mathrm{A})$ at APRT base 346 (exon 4) resulting in substitution of alanine (GCC) by threonine (ACC) at codon 116 (Figure 1); thus, his APRT deficiency was caused by a novel APRT mutation.

APRT deficiency has been noted since 1968 and can be classified as complete deficiency (type I) or partial deficiency (type II) ${ }^{5}$. To date, 2 large population groups with APRT deficiency have been reported. In Iceland, 23 cases homozygous for type I APRT deficiency have been described ${ }^{4}$. Among these cases, 15 of whom had nephrolithiasis, 4 had mild to moderate renal insufficiency and one patient died of uremic complication ${ }^{4}$. In Japan, more than 120 cases with APRT deficiency have been reported ${ }^{6}$ and $78 \%$ were found to be type II. All type II patients carry at least one APRT*J allele with an ATG (methionine) to ACG (threonine) base substitution at amino aicd position $136^{6}$.

Evidently, our case represented a heterozygote mutation $(\mathrm{G} \rightarrow \mathrm{A})$ at

Table 1. Comparison of APRT mutations reported in Japanese, Icelandic, and Chinese populations.

\begin{tabular}{lccc}
\hline Population & Nucleotide & Codon & Exon \\
\hline Japan $1985^{5}$ & 407 & 136 & 5 \\
Iceland $1991^{4}$ & 194 & 65 & 3 \\
China 2008, & 346 & 116 & 4 \\
$\quad$ current report & & & \\
\hline
\end{tabular}
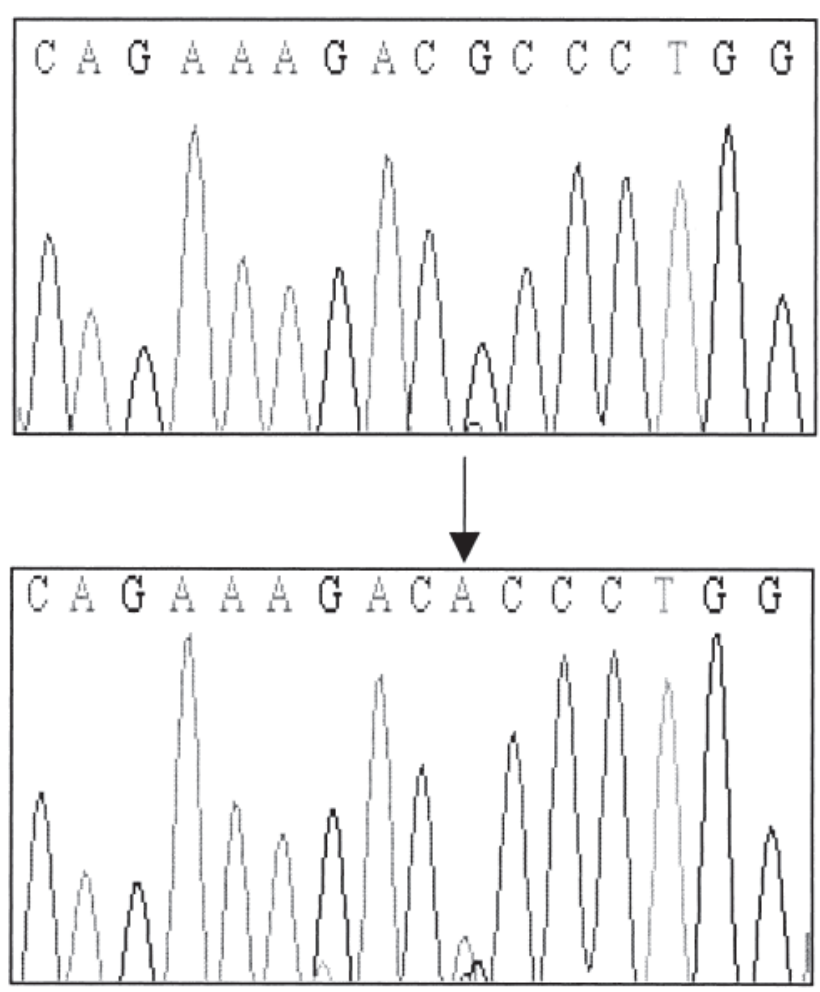

Figure 1. PCR-DNA sequencing revealed a heterozygote mutation $(\mathrm{G} \rightarrow$ A) at APRT base 346 (exon 4) resulting in substitution of alanine (GCC) by threonine (ACC) at codon 116 .

APRT base 346 (exon 4), resulting in alanine (GCC) to threonine (ACC) substitution at codon 116, which is different from the well known APRT*J in Japanese (Table 1). As well, the clinical presentation with early-onset gout in our case was also different from most reports describing presentation of nephrolithiasis or renal failure ${ }^{4,6}$

Whether an APRT mutation is related to gout remains debatable. Kelley, et al reported the first APRT mutation in $1968^{5}$. In 1974, Delbarre, et al found APRT deficiency in persons with gout, but they recognized that purine overproduction was not necessarily caused by the APRT deficien$\mathrm{cy}^{7}$. In 1975, Emmerson, et al described a family with dominant inheritance of APRT deficiency. Although the female proband had gout, they could not confirm a relationship between APRT deficiency and gout ${ }^{8}$. To date, APRT deficiency has been regarded as a risk factor for gout by some Japanese investigators ${ }^{9}$; this has not been confirmed in other populations. In the future, a cohort study of cases with early onset-gout in Chinese or other populations may answer this question.

CHUNG-JEN CHEN, MD, Associate Professor, Division of Rheumatology, Allergy and Immunology, Chang Gung Memorial Hospital-Kaohsiung Medical Center, Chang Gung University College of Medicine, No. 123, Ta-Pei Road, Niao-Sung Hsiang, Kaohsiung Hsein, Taiwan 83301; H. RALPH SCHUMACHER, MD, Professor, University of Pennsylvania, Veterans Affairs Medical Center, Philadelphia, Pennsylvania USA. Address reprint requests to Dr. Chen; E-mail: chungjen@adm.cgmh.org.tw

Supported by Chang Gung Memorial Hospital (CMRP 83014). We thank the patient and his family who agreed to publish this report to benefit patients with early-onset gout to get correct diagnosis and treatment. We thank Ming-Chi Yang for technical assistance. 


\section{REFERENCES}

1. Hung SI, Chung WH, Liou LB, et al. HLA-B*5801 allele as a genetic marker for severe cutaneous adverse reactions caused by allopurinol. Proc Natl Acad Sci USA 2005;102:4134-9.

2. Sakuma R, Nishina T, Kitamura M, Yamanaka H, Kamatani N, Nishioka K. Screening for adenine and hypoxanthine phosphoribosyltransferase deficiencies in human erythrocytes by high-performance liquid chromatography. Clin Chim Acta 1987;170:281-90.

3. Benedetto B, Madden R, Kurbanov A, Braden G, Freeman J, Lipkowitz GS. Adenine phosphoribosyltransferase deficiency and renal allograft dysfunction. Am J Kidney Dis 2001;37:E37.

4. Edvardsson V, Palsson R, Olafsson I, Hjaltadottir G, Laxdal T. Clinical features and genotype of adenine phosphoribosyltransferase deficiency in Iceland. Am J Kidney Dis 2001;8:473-80.

5. Kelley WN, Levy RI, Rosenbloom FM, Henderson JF, Seegmiller JE. Adenine phosphoribosyltransferase deficiency: a previously undescribed genetic defect in man. J Clin Invest 1968;47:2281-9.
6. Kamatani N, Terai C, Kim SY, et al. The origin of the most common mutation of adenine phosphoribosyltransferase among Japanese goes back to a prehistoric era. Hum Genet 1996; 98:596-600

7. Delbarre F, Aucher C, Amor B, de Gery A, Cartier P, Hamet M. Gout with adenine phosphoribosyltransferase deficiency. Biomedicine 1974;21:82-5.

8. Emmerson BT, Gordon RB, Thompson L. Adenine phosphoribosyltransferase deficiency: its inheritance and occurrence in a female with gout and renal disease. Aust NZ J Med 1975;5:440-6.

9. Yamaoka T, Itakura M. Metabolism of purine nucleotides and the production of uric acid [Japanese]. Nippon Rinsho 1996; 54:3188-94.

J Rheumatol 2009;36:5; doi:10.3899/jrheum.081051 